\title{
ELECTRONICS IMPROVEMENTS FOR LOW-LEVEL CONTROL IN THE HUMANOID ROBOT TEO
}

\author{
J. M. Garcia-Haro \\ Higher Polytechnic School, Universidad Francisco de Vitoria, Ctra. Pozuelo-Majadahonda Km 1,800, \\ 28223 Pozuelo de Alarcón, Spain; juanmiguel.garcia@ufv.es \\ E. D. Oña, R. de Santos-Rico, S. Martinez and C. Balaguer \\ System Engineering and Automation Department, University Carlos III, Av de la Universidad 30, 28911 \\ Madrid, Spain; eona@ing.uc3m.es, rasantos@it.uc3m.es, scasa@ing.uc3m.es; balaguer@ing.uc3m.es
}

\begin{abstract}
This work began with developing the first electronic integration to allow the operation of commercial TEO devices pre-selected. However, different problems were discovered during the implementation of the whole set. These were caused by the malfunction of the system and the particular design. For this reason, this paper is focused on the analysis of the first hardware architecture to propose and develop a solution that improves the performance of the joint motor control. Significantly, the detected problems are voltage drops in the power supply signals of some devices, electrical noise coupling or overshoots produced by the switching of the power inverter of the driver, and the electromechanical disconnections of various signals caused by the relative motion between devices. The consequence was that the robot joints control was not robust, preventing them from moving accurately. After the analysis, new interconnection electronics was developed. This electronic has been designed to be more integrated with the mechanic parts, improving subsystems location, and integrating new solutions to reduce the electrical problems. The new electronics have been tested in the humanoid robot arms with good results.
\end{abstract}

Keywords: low-level control, humanoid, electronics.

\section{INTRODUCTION}

A hardware architecture is characterised by its components and the connections between these components. First of all, the connection between the components must have sufficient bandwidth. As the whole architecture shall be able to process data in real-time, the connection as well has to be able to transmit data in real-time [2][15]. Evaluating the needed bandwidth for servo control, it is evident that it can be dealt with one or more typical CAN buses. CAN bus is one of the best options because of its characteristics, i.e. it can transmit messages in real-time. In other words, the message with the highest priority will be delivered within a guaranteed latency time [6][13].
So the different humanoid robots share a peculiarity among them. All of these robots have the same kind of distributed hardware architecture. There are low-level control systems (drivers) which control each joint.

Nevertheless, all of them must work synchronised to control a more complex mechanical system. For this purpose, it is necessary to share data among all the control systems through a high-speed communication system [1].

The RoboticsLab research group at the University Carlos III of Madrid is working with the humanoid robot TEO (Task Environment Operator) (Figure 1). Like the other humanoid robots, TEO has a distributed hardware architecture.

The architecture of TEO is divided into three parts. The first one is the central computer system which consists of two CPUs. The second part is the low-level control system, in which the ISCM8005 driver is used for each joint. And finally, the communication system. CAN bus networks have been selected to share the information among drivers and CPUs.
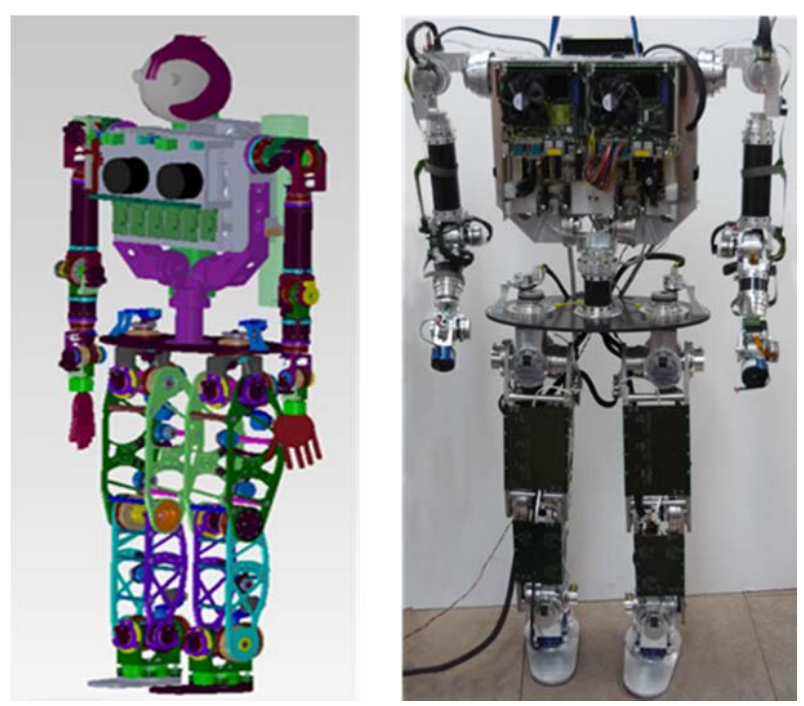

Figure 1: The Humanoid Robot TEO: Whole-Body 3D model representation (left) and real and developed humanoid robot TEO (right). 
The synchronised operation of these parts is possible because the first interconnection electronics or electronics of integration has been developed. This electronics serves to interconnect sensory, power and communication signals from all the control systems and protect the hardware. However, during the setting up of the whole body, different problems caused by the operation of the system and the particularity of the design were discovered. These problems are:

- Brief disconnections between the driver and the motor/encoder affecting the transmission of information.

- Voltage drops causing the shutdown of the devices.

- EMI emissions producing incorrect readings by the driver.

Thus, in this paper, we explain in six parts the development of a new electronics of integration to solve the problems of the first electronic. In section two, we give details about what the hardware architecture and their sub-systems are. In section three and four, the first electronics of integration of the hardware architecture and its problems that have arisen in the low-level control of the joints of the arms are described. In section five, the updated developed integration electronics is described and which tools have been used to improve joint motor control. In the last section, the conclusions are exposed.

\section{TEO HARDWARE ARCHITECTURE}

The RoboticsLab research group at the University Carlos III of Madrid (UC3M) is working on the development of the platform TEO. The purpose is to suit better the needs for using this platform in a human environment, equipping it with a special sensors system, composed of force/torque sensors in the wrists and the feet, together with cameras in the head, and inertial sensors in the chest [9].

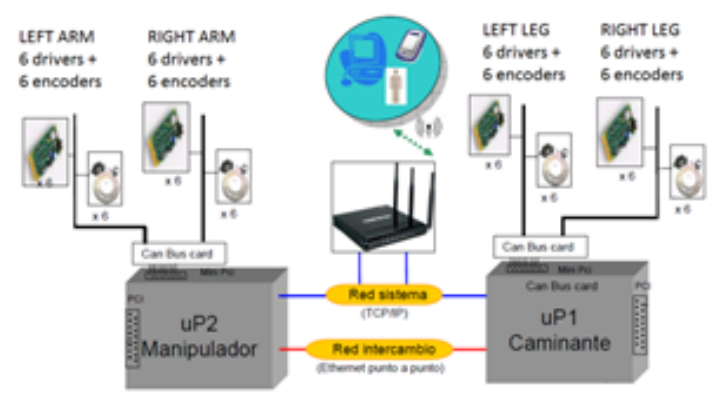

Figure 2: Old TEO hardware architecture.
TEO addresses challenges in the fields of motion, safety, energy efficiency, and power autonomy performance. The mechatronics of this structure has been inspired to perform human adaptable locomotion and achieve complex tasks with humans physically. For this reason, the robot TEO has 28 degrees of freedom [10]: $12 \mathrm{DoF}$ for the locomotion tasks, $12 \mathrm{DoF}$ for the manipulation tasks, 2 DoF for the hip joints and $2 \mathrm{DoF}$ for the neck joints.

The hardware architecture of TEO consists of three sub-systems. The first sub-system is the primary computer system. It has two central CPUs (Intel Core 2 Duo 2 x $2.13 \mathrm{GHz}$ processor), and they are located in the chest. The aim is to divide the tasks between the two CPUs. The first CPU will be responsible for controlling the legs, keeping the balance and generating walks. The second CPU will perform mainly manipulation and grasping tasks with the arms and the trunk.

The second sub-system is communications. On the one hand, the communications between the two main boards or CPUs of the robot are implemented through a high-speed Ethernet connection. In that way, we can achieve speeds up to $1 \mathrm{Gbps}$. On the other hand, a high velocity CAN bus network is used to transmit the sensory and position motor information from and to the drivers [14]. Thus, there are four CAN bus networks (one per each limb).

The third sub-system is the joint motor controller. First of all, the actuators that TEO uses are brushless DC motors from Maxon, generating up to $90.5 \mathrm{mNm}$ of nominal torque. These motors work in combination with strain wave gearings (CSD-series from Harmonic Drive LLC). The ISCM8005 driver is used to control position, velocity and torque. This driver allows controlling different kinds of motors with high current and voltage values. Besides, AMT203-V relative encoder from CUI INC is included to know the relative position and velocity. The drivers are located under the primary computer system, while the motors are situated in their joints (Figure 2).

\section{ELECTRONIC DESIGN OF TEO. V1.0}

The first integration electronics was developed according to the distribution of the central microprocessors, the drivers and the motors. On the one hand, the microprocessors were placed in the chest, and the drivers were under them. On the other hand, the motors were placed along the arms. There were three motors on the shoulder, giving it 3 DoF (Degrees of Freedom) in the sagittal, frontal and axial planes; one on the elbow offering 1 DoF in the frontal plane, and two further on the wrist attributing $2 \mathrm{DoF}$ in the frontal and axial planes (Figure 3). 


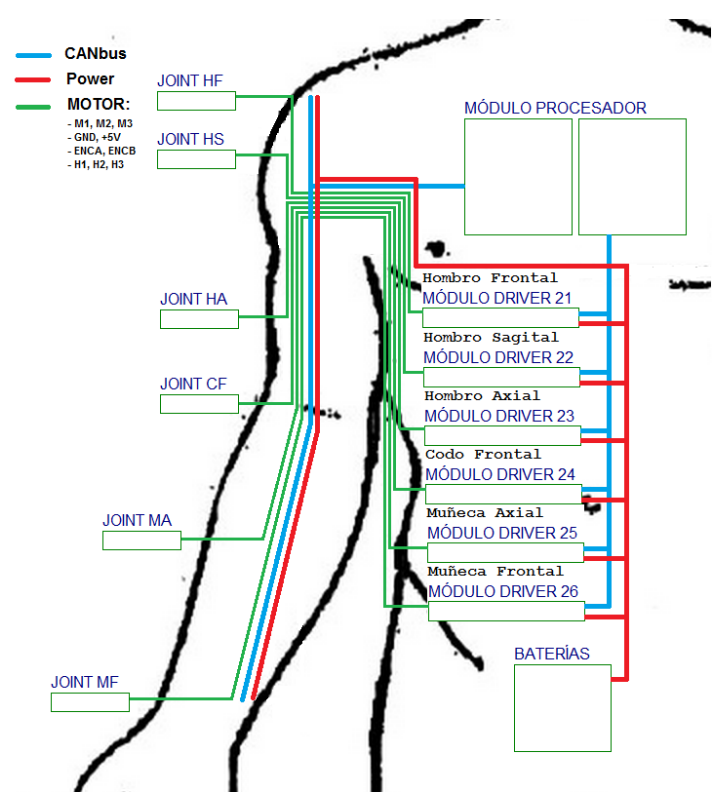

Figure 3: Old TEO hardware distribution.

With this distribution, interconnection electronics should ensure that all systems are physically communicated and integrated. For this purpose, the CAN bus should be interconnected from the microprocessors to the drivers and encoders along the arms (three signals). It also was necessary to wire up from the power supply (positive and negative signals) to supply the drivers and the microprocessors.

Moreover, the drivers should supply their hall sensors and encoders. Thus, drivers should support the entire electrical load. Besides, input sensory information and output control information should be transmitted between the drivers and the joint (motor, hall sensor, and encoder) respectively (eight signals per joint) (Figure 4). In summary, the electronics had more than 50 signals (wires) parallel with a maximum length of $0.5 \mathrm{me}-$ ters which interconnected the sub-systems.

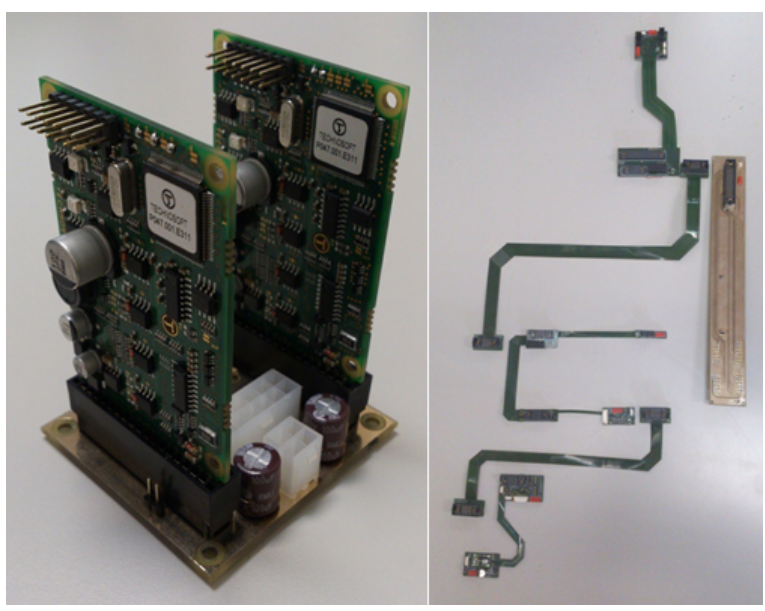

Figure 4: The ISCM8005 driver (left). Interconnection electronics (right).

\section{SETTING UP PROBLEMS IN TEO}

The main operation of the low-level control is based on transmitting position data of each joint from the microprocessor to the drivers. On the other hand, sensory information from the encoder and hall sensors must also send to the drivers. Finally, the drivers must respond to the received information and send it to the motors to control them. For the TEO case, during the system's operation, some issues arose in the first interconnection electronic for controlling the joints. The consequence was that the different signals were not electrically clear and accurate, predominantly sensory and communication signals. It was discovered that this interconnection electronics was not able to protect the system against EMI emissions, voltage drops or brief disconnections. Thus, the joint motor controller failed because the hardware architecture had design problems. These three troubles affected negatively the operation of the robot when the drivers attempted to control the position, velocity or torque of the motor.

\subsection{Brief Disconnections}

The brief disconnections between the motor and the driver are the first problem. These troubles are produced by defective electromechanical contacts. There are two causes. The first one is the relative movement between the electronic control and the motor/encoder. The drivers for the arms are located in the chest, under the motherboards. So there are relative movements concerning the joints in the arms and the drivers. Such movements can cause a faulty contact between the connectors of the interconnection electronics during the operation, causing the loss of the information signal (encoder signals, hall sensor signals, communication signals or power signals). Also, another problem associated is the quality of the connectors. In this case, the connectors used are not very robust, and it has a high tolerance for movements (not kept fixed). So, this feature increases the likelihood of disconnection.

The other cause is produced during the operation of the whole system. During the system's operation, some bending and twisting forces appear due to the synchronised movements of the motors. These forces are transmitted to the skeleton of the robot. Moreover, the interconnection electronics is mechanically coupled to the skeleton. Hence, the electronics also supports bending and twisting forces. The continued application of forces on the electronics produce breaks in the weldings and, therefore, the disconnections of systems. Besides, electronics of integration has two features that foster this undesired state. First, it is divided into several PCBs, and second, the thickness of the PCBs is very fine (to reduce weight). So, the probability that some welding is broken is greater. Finally, if any welding is broken, the system will stop. 


\subsection{Voltage Drops}

The voltage drops are the second problem. This problem arises during the low-level joint motor control when the motors need more energy in some particular situations. These special situations are associated with moments when electricity demand is far greater than the average regular demand. In particular, during acceleration and braking would be the moments when the motors absorb the most current. The motors need to consume more power (more torque) to overcome the forces and inertia opposite the movement (gravity, friction).

On the one hand, the DC brushless Maxon flat motors that TEO uses have three hall sensors powered from 5 volts to 18 volts. On the other hand, the driver can supply 5 volts. So the hall sensors take advantage of this feature to be powered to 5 volts. However, this means that they are working with the minimum allowed voltage.

Furthermore, the driver must support the entire electrical load. It supplies 5 volts to the hall sensors and the encoder too and 36 volts to the motors. So, when there is greater electrical demand, the power supply voltage of the hall sensors drops below 5 volts. At this moment, the hall sensors do not have enough voltage and break down. The system losses information about the position of the rotor, and finally, the low-level control fails.

\subsection{Overshoots}

The overshoot is the third problem, and the ISCM8005 driver produces it. The power electronic of this driver is a typical six-pulse bridge inverter that feeds brushless DC motors with a PWM voltage. The PWM (Pulse Width Modulation) is the technique used to switch the gates in the inverter (Figure 5).

Furthermore, the evolution of power semiconductors has led to the creation of efficient and ever faster transistors. The high switching frequency of electronic transistors used in current converters (usually IGBTs) causes unintended consequences. Some consequences are the increased electromagnetic emissions and the likely impact of surge voltage due to the high values of $\partial V / \partial t$ (rate of voltage change over time) at the terminals of the motors.

On the one hand, the fast-switching speed of semiconductor devices in power inverter leads to self-inflicted voltage transients due to the stray parasitic noise or overshoot [7][4]. Electrical noise in electric machines is caused by the interaction of the fundamental and harmonic flux densities. The electrical noise is generated when the harmonic flux produced is concentrated at a particular frequency, such as the switching frequency.

\section{Pulso de tensión}

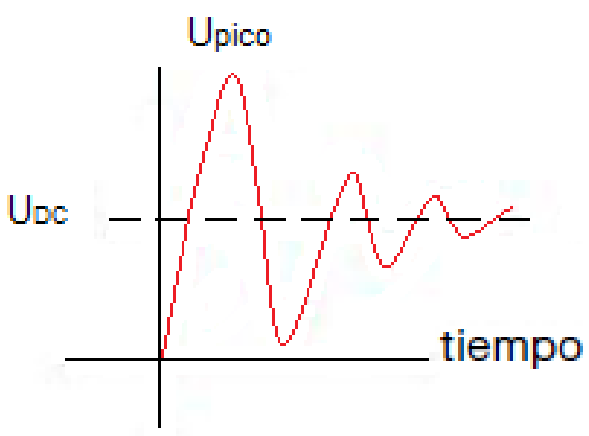

Figure 5: Overshoot's generation during the switching.

On the other hand, the wiring and the motor can be considered a resonant circuit excited by the driver. When the resistors, inductances and capacitors' values are such that the peak voltage exceeds the highest values of the source voltage $(\mathrm{Vdc}=5 \mathrm{~V})$, the circuit response to this excitation is considered an overshoot. The overshoots mainly affect the insulation between spirals, and its value is determined by the "rise time" of the voltage pulse, the cable length, the minimum time between pulses, and the switching frequency [3]. Finally, overshoots' trouble produces electromagnetic emissions coupled to other signals (power, encoder, hall sensors). This one is the main problem that produces incorrect readings by the driver and thus seriously affects the motor control at a low level.

\subsection{Methods for improving electronic}

There are numerous widely methods used to minimise the overshoot of the drivers. The four main methods are a "careful design" of the PCB to minimise parasitic inductance, adding a "bootstrap circuit" for delaying the ignition transistor itself, including a "snubber circuit" for attenuating peak overshoots, or attaching a "circuit CSI" (Common Source Inductance) for delaying the ignition of the transistor itself. The disadvantage to applying these methods is that these methods directly attack the problem at the root. In this case, the overshoot is generated in the three-phase inverter of the driver, which is completely closed to us. The driver is a commercial product which the necessary information and tools are not provided us for being able to apply the methods explained [5][16][8].

Therefore, to keep reusing ISCM8005 drivers, the only solution was to work with the switching frequency of the PWM voltage. The method consisted of decreasing the switching frequency to reduce the rise time and the minimum time between pulses and thus to mitigate or eliminate the overshoot. 

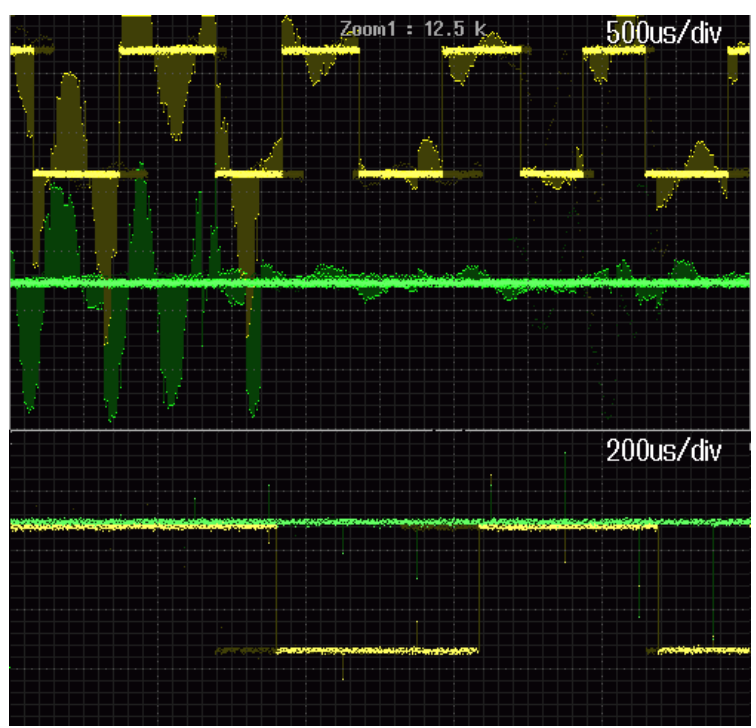

Figure 6: Hall signal with the first interconnection electronics $(100 \mathrm{KHz}$ and $10 \mathrm{KHz})$.

For this, two experiments were prepared. In both experiments, a trapezoidal signal simulating a reference position was applied to the motor. In the first experiment, the switching frequency was $100 \mathrm{KHz}$, and in the second experiment, the frequency was $10 \mathrm{KHz}$.

Figure 6 shows the hall and power signals. The yellow waveform represents the hall signal, and the green one represents the power of the hall sensor. We can see that the overshoot is reduced by lowering the switching frequency. However, this decline is not sufficient, and the problem persists. This result is due to the internal electronics ISCM8005 driver. Figure 7 shows the sensing electronic for the hall sensors signals. This electronic is not very robust. This means that the driver's sensitivity is great when it has to read data from the hall sensors. The high sensitivity allows misinterpreting overshoots generated by the inveeter most likely.

For example, when the hall signal is at a high level and a negative peak is produced, the voltage can be near zero volts, and the driver can interpret the hall signal as low level. Similarly, when the hall signal is at a low level and a positive peak is produced, the voltage can be near 5 volts, and the driver can interpret the hall signal as high level.

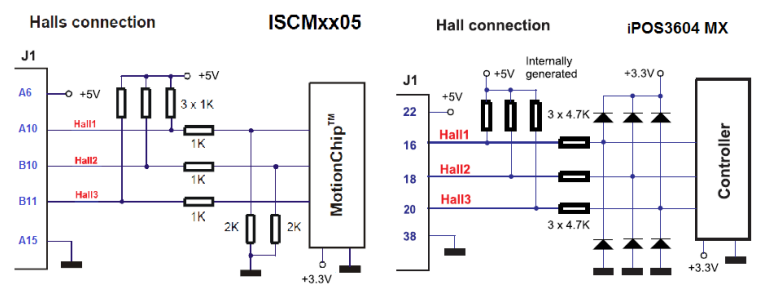

Figure 7: Sensory electronic: ISCM8005 (left), iPOS3604 (right).

\section{TEO V2.0 IMPROVED MECHATRONICS}

In the first version, the interconnection and protection devices were separated in a different board, as figures 3 and 4 show. So this means that each board could have diverse problems. For this reason, is has been developed a single modular electronics which is valid for any joint of the arm.

The main aims in the development of electronic design are three. We agreed that a new controller should be integrated into a small space so that the drivers could be situated along the arms. Thus, the relative movements between the controller and the joint motor would be removed, and the wiring would be reduced. It should also be able to integrate a power system to reduce the electrical load on the driver. Finally, it should have the components and devices needed to reduce overshoots and voltage drops (Fig. 8).

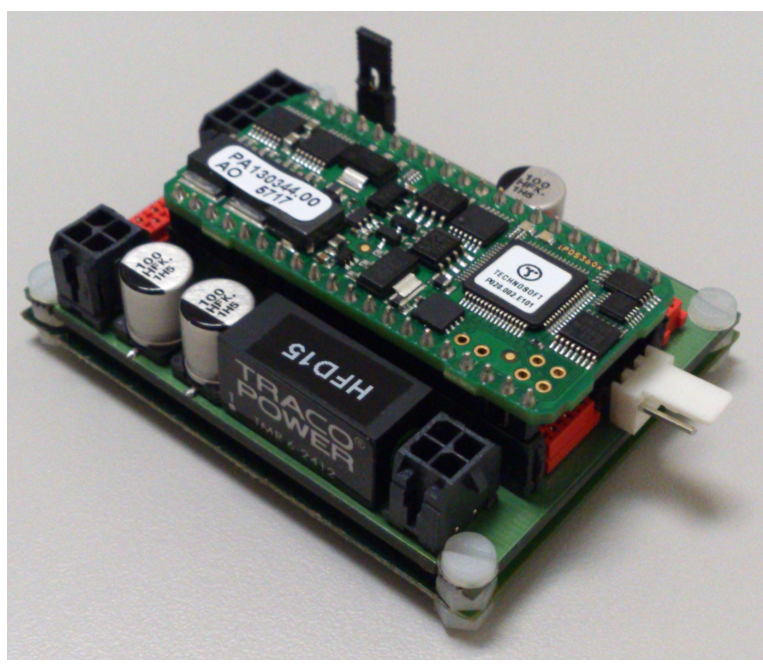

Figure 8: New electronic of integration with the driver.

First of all, the problem of brief disconnection directly depends on the hardware architecture distribution and indirectly depends on the size of the ISCM8005 drivers. The dimensions of these drivers are $79.57 \times 45.62 \times 17.8 \mathrm{~mm}$. Hence, they are pretty big to place them along the arm. For these reasons, we have decided to use another driver with the same power features but smaller than the ISCM8005 driver.

The new driver is from Technosoft too, and it is iPOS3604MX-CAN. It is more intelligent and evolved than the ISCM8005 driver, and its size is $55 \times 26.4 \times 13.1$ $\mathrm{mm}$. Besides, the best advantage of this driver is the type of plug-in that it uses. In the ISCM8005 driver, the plug-in is a card edge connector, and it forces to be connected with the electronics vertically, wasting much space. However, the iPOS3604MX-CAN driver has standard header square pins, which allow being connected horizontally. 
Using the iPOS3604 driver with its horizontal plug-in, we could change the hardware architecture distribution completely. Now, drivers are placed next to the motor. Thus, we achieve firstly prevent relative movements of all joints. The net CANbus and power signals are wired only. So, electromagnetic interference could not be coupled to other signals, which were in parallel with them. Furthermore, we have changed the connectors. Now, the connectors used are more robust, and it has a low tolerance for movements. Therefore, the likelihood of disconnection is very low.

For the problem of the voltage drops, we have added a DC-DC converter module which regulates the input voltage from 36 volts to 12 volts. The goal of this converter module is to divide the electrical loads and avoid voltage drops during the operation. With this converter, the new supply distribution is separated into three parts. One power supply must provide 36 volts to the motor. The electronic of the driver and the hall sensors must be stocked up with 12 volts by a second source. The third power supply, which generates 5 volts inside the driver, must feed the encoder. Thereby, the necessary current is obtained directly from the main power supply (batteries) and not from the driver. Consequently, the electrical loads are divided, and thus, the supply signals are more stable.

Additionally, the integration of the decoupling capacitors also helps to minimise the voltage drops. These decoupling capacitors must be placed as close to the supply inputs as possible. Thereby, when the system demands extra power, the capacitors provide it quickly.

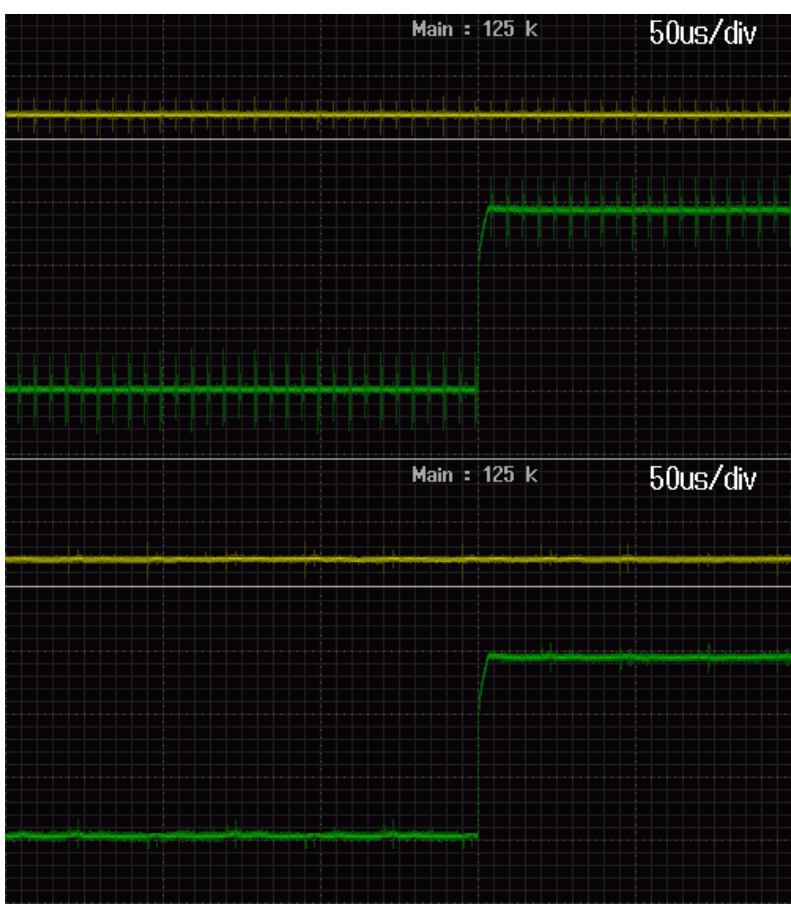

Figure 9: Hall signal with the new electronics of integration $(100 \mathrm{KHz}$ and $40 \mathrm{KHz})$.
At last, the problem of overshoots depends on the driver ISCM8005. The fast-switching speed on the power inverter produces overshoots. Besides, the electronic sensing for the hall signals is not very robust, so the high sensitivity allows misinterpreting overshoots coupled to the hall signals.

The new driver is more evolved. However, the power inverter still generates overshoots, although overshoot intensity is lower than the previous one. The solution to this problem lies in the sensory electronic. This electronics is less susceptible to electromagnetic interference, and therefore the capability to misinterpret the hall signals is lower (Figure 7).

For the prevention of the coupling of overshoot, three methods have been used. The first method is reducing the length of the wires. In this case, with the new distribution, the drivers are situated next to the motor. So the length of the wires to communicate between them is shorter.

The second method is associated with the decoupling capacitors. The electromagnetic interference caused by the driver is shunted through the capacitor (local decoupling), reducing the effect it has on the rest of the circuit [17].

The last method is shielding the signal. For this, the wiring has been shielded with a metallic mesh connected to the ground. Ground planes have been developed in the printed circuit board of the interconnection electronics. This protection technique makes a field, which is able to avoid the coupling of EMI emissions to the signal inside the metallic mesh or the ground plane [11][12].

To test whether improvements are effective, we have reapplied the same two experiments to new electronics. In both experiments, a trapezoidal signal simulating a reference position has been applied to the motor. Now, switching frequencies are $100 \mathrm{KHz}$ and $40 \mathrm{Khz}$.

Figure 9 shows the hall signal (green waveform) and the hall supply signal (green waveform). On the one hand, the hall supply signal still has overshoots, but now the amplitude peaks of the noise are not large enough. Hence, the overshoots do not affect the average voltage of the supply signals. The new power distribution and the addition of protection devices (decoupling capacitors) prevent the voltage drops appearance.

On the other hand, the waveform of the hall signal does not show any electrical noise. The EMI interference's intensity generated by the switching of the power inverter is no longer critical. Therefore, the sensory electronic is able to filter small overshoots, and the driver can obtain correct signals readings from the hall sensors. 


\section{CONCLUSIONS}

In this paper, we have been detected and evaluated the different problems that arose in the first interconnection electronic during the operation of the whole system. Firstly, the brief disconnection because the electromechanical contacts were defective. Secondly, the voltage drops in the hall sensor and encoders because the driver supported the entire electrical load. Finally, the overshoot's coupling to the signals due to switching the power inverter inside the ISCM8005 driver.

To solve these problems, we have defined a new hardware architecture distribution, and we have developed a new modular electronic of integration. The methods and devices, which we have used in the development, are valid. The new distribution has removed all the relative movements between the drivers and motors. The new power distribution has divided the electrical loads. And the new iPOS3604 driver has improved the reading of the sensor signals. So, we have achieved to improve the operation of the low-level control.

\section{Acknowledgement}

This research was funded by the SHARON project, with reference SHARON-CM-UC3M, funded by the Carlos III University of Madrid, and from the RoboCity2030-DIH-CM Madrid Robotics Digital Innovation Hub ("Robotica aplicada a la mejora de la calidad de vida de los ciudadanos, Fase IV"; S2018/NMT-4331), funded by "Programas de Actividades I+D en la Comunidad de Madrid" and cofunded by Structural Funds of the EU.

\section{References}

[1] M. Arbulú and et al. The rh-1 full-size humanoid robot: Design, walking pattern generation and control. Applied Bionics and Biomechanics, 6(34):301-344, 2009.

[2] T. Asfour and et al. Armar-iii: An integrated humanoid platform for sensory-motor control. In Humanoid Robots, 6th IEEE-RAS International Conference on, pages 169-175. IEEE, 2006.

[3] J. Cros and et al. Emi tests on a brushless actuator: comparison of different operation modes. In Fifth European Conference on Power Electronics and Applications, pages 138-143. IET, 1993.

[4] T. G. Habetler and D. M. Divan. Acoustic noise reduction in sinusoidal pwm drives using a randomly modulated carrier. IEEE Transactions on Power Electronics, 6:356-363, 1991.

[5] M. Ingels and M. S. Steyaert. Design strategies and decoupling techniques for reducing the effects of electrical interference in mixed-mode ic's. Solid-State Circuits, IEEE Journal of, 32(7):1136-1141, 1997.

[6] F. Kanehiro, H. Hirukawa, and S. Kajita. Openhrp: Open architecture humanoid robotics platform. The International Journal of Robotics Research, 23(2):155-165, 2004.

[7] Q. Liu and et al. Emi suppression in voltage source converters by utilizing dc-link decoupling capacitors. Power Electronics, IEEE Transactions on, 22(4):1417-1428, 2007.

[8] J. E. Makaran. Gate charge control for mosfet turn-off in pwm motor drives through empirical means. Power Electronics, IEEE Transactions on, 25(5):1339-1350, 2010.

[9] S. Martínez and et al. Teo: Full-size humanoid robot design powered by a fuel cell system. $C y$ bernetics and Systems, 43:163, 2012.

[10] S. Martínez de la Casa Díaz. Human inspired humanoid robots control architecture. 2012.

[11] M. I. Montrose. Printed circuit board design techniques for EMC compliance, volume 1. IEEE press Piscataway, NJ, 1996.

[12] H. W. Ott. Electromagnetic compatibility engineering. John Wiley \& Sons, 2011.

[13] I.-W. Park, J.-Y. Kim, S.-W. Park, and J.-H. Oh. Development of humanoid robot platform khr2. International Journal of Humanoid Robotics, 2:519-536, 2005.

[14] P. Pierro. Stabilizer architecture for humanoid robots collaborating with humans. 2012.

[15] K. Regenstein and et al. Designing a computer architecture for the humanoid robot armar-iii. In Proceedings of French-German Workshop on Humanoid and Legged Robots, 2006.

[16] L. Rossetto, S. Buso, and G. Spiazzi. Conducted emi issues in a 600-w single-phase boost pfc design. Industry Applications, IEEE Transactions on, 36(2):578-585, 2000.

[17] F. Zare. Emi issues in modern power electronic systems. The IEEE EMC Society Newsletters, (221):53-58, 2009.

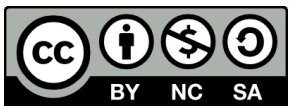

(C) 2021 by the authors. Submitted for possible open access publication under the terms and conditions of the Creative Commons Attribution CC BY-NCSA 4.0 license (https://creativecommons.org/licenses/by-ncsa/4.0/deed.es). 